\title{
Estudio de un neo-epítope para detección de anticuerpos antitransglutaminasa en enfermedad celíaca
}

\section{Validation of a new antigen for detection of anti-transglutaminase antibodies in celiac disease}

\author{
Sandra Verbeke ${ }^{1,2^{*}}$, Nancy Gutierrez ${ }^{2}$, Miguel Catalán' ${ }^{1}$ Andrea Canals ${ }^{3}$
}

\section{Resumen}

Introducción: La enfermedad celíaca (EC) es una patología autoinmune, que se desarrolla a cualquier edad en personas genéticamente susceptibles, y cuyo órgano diana principal es el intestino delgado. La diversidad en las formas de presentación actualmente conocidas implica un desafío permanente para el laboratorio, que debe ofrecer nuevas técnicas, cada vez más sensibles y específicas, para detectar de manera eficiente los autoanticuerpos específicos para el diagnóstico y seguimiento de estos pacientes. Nuestro objetivo fue evaluar la sensibilidad y especificidad de un nuevo antígeno para la detección de anticuerpos anti-transglutaminasa compuesto por transglutaminasa tisular unida covalentemente a péptidos deamidados de gliadina (neo-epítope) en pacientes con sospecha de EC, con biopsia duodenal como gold standard. Como objetivo secundario nos propusimos evaluar la sensibilidad y especificidad del antígeno convencional, transglutaminasa humana recombinante, para el mismo grupo de estudio. Metodología: Se realizó un estudio prospectivo, analizando muestras de pacientes con sospecha de EC o en seguimiento de dicha patología, en las que se estudiaron los anticuerpos anti-transglutaminasa con antígeno neo- epítope, y con antígeno transglutaminasa recombinante humana. Se determinó sensibilidad, especificidad, VPP, VPN y coeficiente de concordancia Kappa. Resultados: Se procesaron 56 muestras, incluidas en un período de 5 meses. La sensibilidad (100\%) y especificidad (92,3\%) obtenidas con la técnica de neo-epítope, en relación a la biopsia (gold standard), fue mayor que con la técnica transglutaminasa humana recombinante (88,3\% y 78,9\% respectivamente). La técnica con neo-epítope proporcionó un menor número de resultados en la "zona de indeterminación". Conclusiones: Nuestros resultados concuer-dan con otros autores, ya que neo-epítope detecta con mayor sensibilidad y especificidad aquellos pacientes con diferente situación de presentación y transgresores de la dieta libre de gluten, quienes pueden presentar serología negativa o débilmente positiva con transglutamiasa humana recombinante. La nueva técnica neo-epítope constituiría una mejor herramienta para la pesquisa diagnóstica y de seguimiento en pacientes con EC.

Palabras clave: enfermedad celíaca; antitransglutaminasa; autoanticuerpos; autoinmunidad.

Abstract

Introduction: Celiac disease (CD) is an autoimmune disease, which develops at any age in genetically susceptible people, and whose main target organ is the small intestine. The diversity in the currently known forms of presentation implies a permanent challenge for the laboratory, which must offer new techniques, increasingly sensitive and specific, to efficiently detect the specific autoantibodies that collaborate in the diagnosis and follow-up of these patients. Our main objective was to evaluate the sensitivity and specificity of a new sensitizing antigen for the detection of anti-transglutaminase antibodies composed of tissue transglutaminase covalently linked to deamidated gliadin peptides (neo-epitope) in patients with suspected CD, with duodenal biopsy as the gold standard. As a secondary objective, we set out to evaluate the sensitivity and specificity of the conventional antigen, recombinant human transglutaminase, for the same study group. Methodology: A prospective study was carried out, including samples from patients with suspected CD or in follow-up of said pathology, in which anti-transglutaminase antibodies were studied with neo-epitope antigen, and with human recombinant transglutaminase antigen. Sensitivity, specificity, PPV, NPV and Kappa coefficient of concordance were determined. Results: 56 samples were processed, included in a period of 5 months. The sensitivity and specificity obtained with the neo-epitope technique (S: 100\% - E: 92.3\%), in relation to the biopsy (gold standard), was higher than with the recombinant human transglutaminase technique

(1) Sección Inmunología, Laboratorio, Clínica Santa María.

(2) Escuela de Tecnología médica, Universidad Santo Tomás.

(3) Dirección Académica, Clínica Santa María. Escuela de Salud Pública, Universidad de Chile

*Autor de correspondencia: sverbeke@vtr.net 
(S: 88.3\% - E: 78.9\%). The neo-epitope technique provided fewer results in the "zone of indeterminacy". Conclusions: Our results agree with other authors, since the neo-epitope detects with greater sensitivity and specificity those patients with different presentation situations and transgressors of the gluten-free diet, who can present negative or weakly positive serology with recombinant human transglutaminase. The new neo-epitope technique would constitute a better tool for diagnostic and follow-up research in patients with CD.

Keywords: celiac disease; antitranglutaminase; autoimmunity; autoantibodies.

Fecha de envío: 21 de enero de 2020 - Fecha de aceptación: 3 de septiembre de 2020

\section{Introducción}

La enfermedad celíaca (EC) es una patología multifactorial y multisis-témica que implica considerar una variada gama de presentaciones clínicas posibles. Puede confundirse con patologías alternativas, tener períodos latentes, presentarse tanto en niños como en adultos y quizás no se le incorpora con la suficiente frecuencia entre los diagnósticos diferenciales en pacientes de presentación no clásica. Todas estas particularidades han impulsado la búsqueda de herramientas diagnósticas por largo del tiempo (Torsten et al, 2010; Volta \& Villanacci, 2011).

Dieterich et al. (1997) identificaron la transglutaminasa tisular (TGt) como el autoantígeno blanco de la EC, lo que significó un gran avance para la pesquisa serológica. Posteriormente, se demostró que la TGt era el antígeno específico de la prueba de anticuerpos anti endomisio (EMA) y que era expresado por los fibroblastos componentes del endomisio (Dieterich et al., 1997; Caja et al., 2011). Esta enzima interviene en la deamidación, siendo la gliadina uno de sus sustratos. En el paciente con EC, el mecanismo de lesión, de naturaleza autoinmune, se produce por el "desconocimiento" de los antígenos que se originan durante el proceso fisiológico de deamidación de la gliadina por la enzima TGt, ya que este proceso incrementa la inmunogenicidad de esta molécula, formándose auto anticuerpos anti-transglutaminasa, autoanticuerpos anti-péptidos deamidados de gliadina (DPG) y autoanticuerpos anti- transglutaminasa tisular covalentemente unida a péptidos deamidados de gliadina (Molberg et al., 1998; Torsten M et al., 2010; Volta \& Villanacci, 2011).

Debido a la gran variabilidad en sus presentaciones clínicas y sin-tomatología extraintestinal con que puede presentarse a cualquier edad esta patología, las sociedades médicas (ESPGHAN -Sociedad Europea de Gastroenterología y Nutrición Pediátrica, NASPGHAN - Sociedad Norteamericana de Gastroenterología Pediátrica, He-patología y Nutrición, Sociedad Británica de gastroenterología y Nutrición, ACG o Colegio Americano de Gastroenterología) se reúnen periódicamente para revisar y establecer criterios y definiciones por consenso para el diagnóstico de EC. Si bien en los últimos años ha habido una tendencia a plantear el diagnóstico prescindiendo de la biopsia, bajo condiciones estrictas (niños y adolescentes con síntomas sugestivos de EC con anti transglutaminasa $\lg A>10$ veces su valor de corte, $y$ anti-endomisio positivo en una segunda muestra $y$ presencia de HLA DQ2/DQ8), es bien conocido que la determinación de los anticuerpos anti-tranglutaminasa no está estandarizada, y si bien la mayoría de los test comerciales muestran una elevada precisión, sobre todo a títulos altos, se han observado variaciones entre diferentes test y entre laboratorios usando el mismo test en niveles de anticuerpos anti-transglutaminasa leves-moderados. Es por ello que las guías internacionales confirman que los anticuerpos anti-transglutaminasa $\lg \mathrm{A}$ deben determinarse en primer lugar en el estudio de la EC junto con la cuantificación de IgA sérica, y que el estudio histológico posterior confirma en diagnóstico, siendo aún considerado como gold standard y pilar fundamental del diagnóstico de EC, lo que también se indica en las guías de nuestro medio (Husby et al., 2012; MINSAL 2015; Husby et al., 2020).

Existe un grupo de pacientes silentes y/o latentes, que no son diagnosticados con facilidad debido a pruebas no concluyentes, síntomas vagos o pacientes ya diagnosticados en los que no se logra detectar la transgresión de la dieta libre de gluten a través de las pruebas serológicas existentes. Además, otras enfermedades del sistema digestivo pueden dar falsos positivos de serología para EC, confundiendo el diagnóstico y llevando incluso a procedimientos innecesarios. Por otra parte, es necesario poder distinguir la EC de otras patologías asociadas al gluten, como la"sensibilidad al gluten no celíaca" y la "alergia al gluten" (McCabe et al., 2012; Fassano \& Catassi, 2012; Pietzak, 2012; Sapone, 2012).

Con la finalidad de contar con una prueba serológica que proporcione una mejor correlación con las situaciones complejas y las condiciones clínicas descritas anteriormente, ha surgido un nuevo antígeno para la prueba de enzimoinmunoanálisis (ELISA) que combina los péptidos transglutaminasa + gliadina, obtenidos como el complejo proteico que se produce en condiciones fisiológicas in vivo, y se ha denominado"neo-complejo tTg" (TGt-DGP) (AESKU-LISA tTg-A New Generation ${ }^{\circledR}$ ) (Torsten et al., 2010; Rozenberg, 2012).

Este neo-epítope intenta reflejar el momento en que la enzima captura al péptido de gliadina para deamidarlo y cuyo 
mecanismo está aún bajo investigación. En pacientes con EC, induce a la formación de autoanticuerpos anti tTg neo-epitope contra las diferentes partes de este grupo proteico y utilizado en técnicas de ELISA, podría detectar un grupo más amplio de au-toanticuerpos aumentando la sensibilidad del ensayo y siendo útil en aquellos pacientes "problema" donde la serología es negativa. Considerando la importancia de un diagnóstico precoz por lo que un paciente con EC puede sufrir al no ser introducido en una dieta sin gluten a tiempo, la idea es "bajar la línea y aumentar la punta del iceberg"tal como lo expresa Lerner (2014), dando un nuevo enfoque al algoritmo diagnóstico, en el cual el neo-epítope tiene un rol preponderante.

Por tanto, nuestro objetivo principal fue evaluar la sensibilidad y espe-cificidad de este nuevo antígeno (neo-epítope) para la detección de anticuerpos anti-transglutaminasa en pacientes con sospecha de EC, considerando como técnica de referencia la biopsia duodenal. Como objetivo secundario nos propusimos evaluar la sensibilidad y especificidad del antígeno convencional, transglu-taminasa humana recombinante, en relación a la biopsia y para el mismo grupo de estudio.

\section{Materiales y métodos}

Pacientes: se realizó un estudio prospectivo de 5 meses de dura-ción (febrero a junio de 2013) en el que se incluyeron 56 pacientes con sospecha y/o en seguimiento para EC, quienes aceptaron, con consentimiento informado, par-ticipar voluntariamente; en el caso de menores de edad, fueron sus tutores quienes firmaron dicha autorización. Se les tomó una muestra de sangre con indicación de ayuno sólido de al menos 3 horas (muestras no hemolizadas, no lipémicas). Las muestras se codificaron y conservaron a $-20^{\circ} \mathrm{C}$ hasta su procesamiento. Todos los pacientes estudiados tenían biopsia duodenal cuyo resultado se consideró referencia (gold standard) para la realización de este estudio. Además, todos los pacientes incluidos tenían cuantificación de lgA sérica en los rangos de referencia.

Este estudio fue aprobado por el Comité de Ética de la institución (código interno del estudio: 060802-13, 8/02/2013, Santiago, Chile) y se obtuvo consentimiento informado para cada paciente incor-porado al estudio, y cuyas muestras se utilizaron en este ensayo.

Metodología: para este estudio se utilizaron kits para detección de anticuerpos anti-TGt AESKULISA tTg-A New Generation ${ }^{\circledR}$ (Neo-epítope conformado por transglutaminasa tisular + péptidos deamidados de gliadina) y ELISA IMMCO Immulisa anti TGt IgA ${ }^{\oplus}$ (transglutaminasa tisular recombinante humana). El valor de corte y el rango de indeterminación considerado en cada caso, y otorgado por el fabricante fue: AESKU >18 U/mL (Indeterminado: 12 a $18 \mathrm{U} /$ $\mathrm{mL}$ ) y para IMMCO > $25 \mathrm{U} / \mathrm{mL}$ (Indeterminado: 20 a $25 \mathrm{U} / \mathrm{mL}$ ). Se testearon por separado y con ambos kits, todas las muestras con la metodología de ELISA, en un analizador automático de ELISA para placas de micropocillos Triturus Grifols ${ }^{\circledast}$.

Estudio estadístico: se realizaron análisis descriptivos de los resultados de ambas técnicas mediante distribuciones de frecuen-cias. Se calcularon sensibilidad, especificidad, valores predictivos positivo y negativo, y coeficiente de concordancia Kappa, con sus respectivos intervalos de confianza. Además, se calculó la razón de verosimilitud positiva y se comparó la proporción de falsos positivos mediante el test de Z para proporciones.

Se calculó la potencia del tamaño de muestra utilizado $(n=56)$ para la detección de una sensibilidad de $99 \%$ dada la hipótesis de nulidad: Sensibilidad $=90 \%$, obteniendo una potencia de $94,7 \%$. Todos los análisis se realizaron con el software Stata 13.

\section{Resultados}

Durante el período febrero a junio 2013 se incluyeron en el estudio 56 muestras de pacientes con las condiciones ya descritas.

Según la situación, se estudiaron 48 pacientes por sospecha diagnóstica de EC, de los cuales 10 resultaron positivos para dicha patología, con biopsia concordante, y 38 fueron negativos, presentando otros diagnósticos y biopsia negativa. Además, 8 eran pacientes con EC en seguimiento de su dieta libre de gluten. El rango de edades fue de 1 a 69 años; 7 fueron niños (1 a 9 años) y 49 adultos (19 a 69 años), 36 de sexo femenino y 20 masculino.

a) Sensibilidad, especificidad y concordancia: se calculó la sensibilidad, especificidad, valores predictivos positivo y negativo, índice de concordancia Kappa y razón de verosimilitud positiva para ambas técnicas en relación a la biopsia duodenal (técnica diagnóstica de referencia). En la Tabla 1 pueden observarse los resultados tanto para el antígeno neo-epítope (anti-TGt-DGP - AESKULISA tTg-A New Generation ${ }^{\circledR}$ ) como para el antígeno transglutaminasa recombinante humana (anti-TGt IMMCO ${ }^{\circledR}$ ). Según el valor del índice de Kappa obtenido, se observa que la técnica realizada con neo-epítope mostró un alto índice de concordancia Kappa en relación a la biopsia. El antígeno TGt recombinante humana obtuvo un índice de concordancia moderado. También se observaron diferencias a favor del antígeno neo-epítope en cuanto a sensibilidad, especificidad, valores predictivos y razón de verosimilitud positiva. Esta última indica cuántas veces más probables es obtener un resultado positivo en un paciente con biopsia positiva que en uno con biopsia negativa, y se observa que esta razón es mayor para la técnica que utiliza neo-epítope que para la que utiliza transglutaminasa humana recombinante. 
Tabla 1: sensibilidad, especificidad y concordancia versus biopsia de antígenos neo-epítope y transglutaminasa humana recombinante.

\begin{tabular}{|c|c|c|c|c|}
\hline & \multicolumn{2}{|c|}{$\begin{array}{l}\text { Transglutaminasa } \\
\text { recombinante humana }\end{array}$} & \multicolumn{2}{|c|}{ neo-epítope } \\
\hline & $\%$ & IC 95\% & $\%$ & IC 95\% \\
\hline Sensibilidad & 88,2 & $(63,6-98,5)$ & 100 & $(80,5-100)$ \\
\hline Especificidad & 76,9 & $(60,7-88,9)$ & 92,3 & $(79,1-98,4)$ \\
\hline VPP & 62,5 & $(40,6-81,2)$ & 85,0 & $(62,1-96,8)$ \\
\hline VPN & 93,8 & $(79,2-99,2)$ & 100 & $(90,3-100)$ \\
\hline Índice Kappa & 0,58 & $(0,37-0,80)$ & 0,88 & $(0,75-1,00)$ \\
\hline Concordancia & \multicolumn{2}{|c|}{ Moderada } & \multicolumn{2}{|l|}{ Alta } \\
\hline RVP* & \multicolumn{2}{|c|}{3,82} & \multicolumn{2}{|l|}{12,98} \\
\hline
\end{tabular}

${ }^{*} \mathrm{RVP}=$ Razón de verosimilitud positiva = Sensibilidad/(1-especificidad), $\mathrm{VPP}=$ valor predictivo positivo, $\mathrm{VPN}=$ valor predictivo negativo. b) Falsos positivos: la proporción de falsos positivos, en relación a la biopsia, y considerando como valores de corte para AESKU >18 $\mathrm{U} / \mathrm{mL}$ y para IMMCO $>25 \mathrm{U} / \mathrm{mL}$, fue mayor en el caso de antígeno humano recombinante $(7,1 \%=4 / 56)$ que para neo-epítope $(3,6 \%$ $=2 / 56$ ), pero esta diferencia no resultó significativa (test de $Z$ para comparación de proporciones, $\mathrm{p}=0,201>0,05$ ).

En la Tabla 2 se muestran los pacientes que, con respecto a la biopsia, mostraron resultados falsos positivos, e indeterminados, en cada caso.

Un análisis adicional que se incluyó en este estudio fue considerar los resultados inferiores a los valores de corte, y dentro del rango "Indeterminado" ("zona de indeterminación" de las técnicas) pro-puesto por el fabricante para ambos antígenos: para Neo-epitope, 12 -18 $\mathrm{U} / \mathrm{mL}$ y en el caso tTG humana recombinante se consideró 20 - 25 $\mathrm{U} / \mathrm{mL}$. La proporción de "Indeterminados" para estos rangos, fue de 5,4\% (3/56) para neo-epítope y de 16,1\% (9/56) para tTG humana recombinante. Al realizar el test de Z para comparar las proporciones, se obtuvo una diferencia significativa $(p=0,0334)$. Esto mantuvo la proporción de los Indeterminados para tTG humana recombinante, mayor que para neo-epítope.

Tabla 2: Resultados falsos positivos de ambas técnicas anti TtG en pacientes con biopsia normal.

\begin{tabular}{ccccccc}
\hline No paciente & Sexo & Edad & tTG humana recombinante $(\mathbf{U} / \mathbf{m L})$ & Resultado & Neo-epítope (U/mL) & Resultado \\
\hline 95 & F & 20 & 20,00 & Indeterminado & 1,15 & Negativo \\
54 & M & 50 & 22,00 & Indeterminado & 2,13 & Negativo \\
50 & M & 61 & 23,40 & Indeterminado & $\mathbf{6 3 , 8 0}$ & Positivo \\
61 & F & 43 & 24,20 & Indeterminado & 2,57 & Negativo \\
10 & M & 69 & 25,00 & Indeterminado & 1,35 & Negativo \\
108 & M & 33 & $\mathbf{2 7 , 7 0}$ & Positivo & 3,16 & Negativo \\
85 & F & 20 & $\mathbf{3 3 , 5 0}$ & Positivo & 1,67 & Negativo \\
113 & F & 44 & $\mathbf{1 2 2 , 0 0}$ & Positivo & 2,29 & Negativo \\
114 & F & 44 & $\mathbf{1 2 6 , 0 0}$ & Negativo & 18,00 & Negativo \\
25 & F & 32 & 6,15 & Negativo & $\mathbf{5 1 , 6}$ & Indeterminado \\
101 & M & 61 & 13 & Positivo & .
\end{tabular}

\section{c) Pacientes con EC en control de dieta libre de gluten}

De los pacientes con EC, que se encontraban en control de la dieta libre de gluten ( $\mathbf{n = 8}$ ), 2 de ellos (muestras $\mathrm{N}^{\circ} 4$ y $\mathrm{N}^{\circ} 56$, ver Tabla 3 ) referían cumplir estrictamente la misma y tanto la biopsia como los resultados obtenidos con ambos antígenos fueron negativos, por lo que se podría inferir un cumplimiento estricto sin transgresiones. Otros 2 pacientes ( $\mathrm{N}^{\circ} 16$ y 64) referían explícitamente el no cumplimiento de la dieta sin gluten y en este caso ambas técnicas fueron capaces de detectar dichas transgresiones, con biopsias concordantes, aunque cabe destacar que neo-epítope arrojó valores más resolutivos. De los 4 restantes que manifestaban el cumplimiento estricto de la dieta, la técnica de neo-epítope detectó anticuerpos anti-TGt positivos en todos los casos, lo que podría indicar la transgresión involuntaria de la dieta en estos pacientes; el antígeno transglutaminasa humana recombinante detectó anticuerpos positivos sólo en 3 de ellos. Todos los resultados comentados anteriormente quedan resumidos en la Tabla 3. 
Verbeke et al.

Tabla 3: Pacientes con enfermedad celíaca en control de dieta libre de gluten

\begin{tabular}{|c|c|c|c|c|c|}
\hline Paciente & $\begin{array}{l}\text { tTg humana recombinante } \\
\qquad(\mathrm{U} / \mathrm{mL})\end{array}$ & Resultado & Neo-epitope (U/mL) & Resultado & Dieta sin gluten \\
\hline 56 & 4,85 & Negativo & 1,0 & Negativo & Sí \\
\hline 4 & 5,34 & Negativo & 1,09 & Negativo & $\mathrm{Si}$ \\
\hline 16 & 73,5 & Positivo & 300 & Positivo & Refiere No \\
\hline 64 & 160 & Positivo & 300 & Positivo & Refiere No \\
\hline 49 & 61,9 & Positivo & 277 & Positivo & Sí ?? \\
\hline 112 & 5,73 & Negativo & 134 & Positivo & Sí ?? \\
\hline 1 & 81,8 & Positivo & 229 & Positivo & Si ?? \\
\hline 55 & 101 & Positivo & 300 & Positivo & Sí ?? \\
\hline
\end{tabular}

\section{Discusión}

Debido a la diversidad de patologías relacionadas con el gluten, los grupos de investigación se encuentran en permanente búsqueda de alternativas que permitan la diferenciación diagnóstica, con la intención de contar con nuevas técnicas que detecten con alta sensibilidad y especificidad los marcadores serológicos característicos de la EC. Estas técnicas, tal como lo afirman otros autores (Lerner et al., 2019), deberían detectar tempranamente las diferentes situaciones que se presentan en la EC (diagnóstico, seguimiento, transgresiones), y lograr una buena resolución de resultados tanto positivos como negativos y disminuir la "zona de indeterminación" o de incerti-dumbre, con valores cercanos al corte, que tanto desorientan al profesional clínico. Es por ello que diversos autores se esfuerzan por reproducir el antígeno que da lugar a la cascada autoinmune y la situación fisiopatológica de la EC que culmina en la síntesis de los autoanticuerpos que componen la herramienta serológica de ayuda diagnóstica (Torsten et al., 2010; Choung et al., 2019).

Los resultados obtenidos en este estudio muestran que la técnica neo-epítope presentó altos índices de concordancia en relación a la biopsia. Tanto la sensibilidad como la especificidad obtenidas con el neo-epitope fueron superiores a las obtenidas con el antígeno convencional transglutaminasa tisular recombinante humana. Estos resultados podrían deberse a que el nuevo antí-geno sensibilizante (neo-epítope) expone 3 epítopes diferentes:

TGt, DPG y el complejo formado por ambos en el momento de la deamidación, lo que aumentaría las posibilidades de pesquisa del grupo que conforman los diferentes autoanticuerpos originados en los pacientes celíacos. Nuestros resultados, además, concuerdan con lo publicado por otros autores, quienes evaluaron varios kits existentes en el mercado y es importante destacar dicha concordan-cia, a pesar de haber contado con un número de muestras menor que los estudios internacionales publicados (Volta \& Villanacci, 2011; Fassano \& Catassi, 2012; Porcelli et al., 2016). Localmente, este antígeno fue incluido en las "Recomendaciones para serología de enfermedad celíaca" (Instituto de salud pública, 2016), como antígeno emergente y marcador de apoyo diagnóstico de reciente introducción.

Aunque esta diferencia no fue estadísticamente significativa, lo que indicaría que la proporción de falsos positivos no sería diferente entre neo-epitope y translgutaminasa recombinante, es importante destacar que con el antígeno neo-epítope se obtuvo un 3,6\% de resultados falsos positivos, en relación a los resultados de la biopsia; para el antígeno TGt humana recombinante se obtuvo un 7,1\%. Cabe comentar, que, si bien llama la atención la menor tendencia de falsos positivos del neo-epitope, quizá un estudio genético HLA para EC podría colaborar para establecer si estos resultados pueden considerarse realmente como falsos positivos, ya que podríamos estar frente a un paciente latente o potencial. Si bien, y tal como lo indican las guías internacionales, el estudio genético HLA no es necesario para reforzar un diagnóstico que ya está bien establecido en base a los resultados de las demás pruebas, posee un elevado valor predictivo negativo que des-carta la enfermedad casi con plena certeza cuando no existeninguna de las variantes proteicas HLA asociadas (DQ2, DQ8 o uno de los alelos de la proteína DQ2: DQA $1{ }^{*} 05$ o DQB $1{ }^{*} 02$ ) (Husby et al., 2020).

Un aspecto importante para nosotros como laboratoristas es la necesidad de una buena resolución de valores, con resultados lo más alejados de la zona de corte de la técnica. Con los resultados obtenidos en este estudio con neo-epitope observamos que la 
utilización de la técnica de neo-epítope, llevaría a una significativa reducción de la "zona de indeterminación" o de incertidumbre, siendo más resolutiva que la técnica TGt humana recombinante. Este análisis se incluyó teniendo en cuenta que el médico solici-tante al recibir un resultado "indeterminado", podría considerarlo como "sospechoso". Es importante destacar que los pacientes que presentaron valores indeterminados presentaron biopsias duodenales negativas para EC.

En relación a la utilidad del antígeno neo-epítope para la sero-logía diagnóstica de EC, éste neoantígeno mostró una mayor sensibilidad que el antígeno TGt humana recombinante. En este estudio, la nueva técnica neo-epítope detectó un mayor número de pacientes con transgresión voluntaria o involuntaria de la dieta, o en seguimiento de su dieta desde el momento de diagnóstico, lo que podría inferir su capacidad para detectar que la mucosa aún no ha normalizado su arquitectura a pesar de la dieta libre de gluten. Silvester et al. (2017) demostraron que los marcado-res habituales no eran buenos indicadores de la normalización histológica, y quizá se podría inferir que neo-epítope tiene una mejor correlación con la mejoría o mejor aún con la detección de mucosa aún con daño histológico, aunque el paciente esté en dieta libre de gluten (Lerner et al., 2016). Es importante recordar lo relevante que resulta para el paciente el cumplimiento de la dieta sin gluten de manera rigurosa y la importancia de contar con una técnica altamente sensible, que sea capaz de detectar mínimas transgresiones para alertar sobre las mismas y permitir al clínico la búsqueda junto al paciente de los posibles alimentos que están provocando dicha transgresión.

\section{Conclusiones}

Consideramos este estudio como preliminar para la realización de uno posterior, con un número mayor de muestras, el cual nos permitiría obtener una mayor variedad de datos en apoyo de la utilidad de este marcador emergente.

La técnica neoepítope presentaría una utilidad según la situación del paciente, es decir diagnóstico, seguimiento y/o transgresión de la dieta, quienes por lo general presentan serología negativa o débilmente positiva.

Además, presentó una "mínima zona de indeterminación" en relación al antígeno humano recombinante, lo que apoya la hipótesis de que el antígeno neoepítope podría resultar una mejor herramienta diagnóstica. Además, la sensibilidad, especificidad, valores predictivos y concordancia obtenidas con neoepítope en relación a la biopsia (técnica diagnóstica de referencia) fueron mayores que con TGt humana recombinante.

\section{Fuentes de financiamiento}

Agradecemos a la empresa Grifols Chile, por donar los kits AES-KULISA tTg-A New Generation ${ }^{\circledR}$ con que se realizó este estudio, lo que permitió además obtener la Tesis de grado de una alumna de Tecnología médica.

\section{Contribuciones y conflictos declarados por los autores}

Aparte de lo ya comentado, los autores declaran no tener conflictos de interés.

\section{Referencias}

Caja S, Mäki M, Kaukinen K. \& Lindfors K (2011). Antibodies in Celiac Disease: Implications Beyond Diagnostics. Cellular and Molecular Immunology 8, 103-9.

Choung R, Rostamkolaei S, Ju j, Marietta E, Van Dyke C, Rajasekaran J, et al. (2019). Synthetic Neoepitopes of the TransglutaminaseDeamidated Gliadin Complex as Biomarkers for Diagnosing and Monitoring Celiac Disease. Gastroenterology 156, 582-91.

Dieterich W, Ehnis T, Bauer M, Donner P, Volta U, Riechen E, et al. (1997). Identification of tissue transglutaminase as the autoantigen of celiac disease. Nature Medicine 3, 797-801.

Fasano A \& Catassi C. (2012) Celiac disease. New England Journal Medicine 367, 2419-26.

Husby S. et al. (2012). European Society for Pediatric Gastroente-rology, Hepatology, and Nutrition guidelines for the diagnosis of coeliac disease. J Pediatr Gastroenterol Nutr 54, 136-60

Husby S, Koletzko S, Korponay-Szabó I, Kurppa K, Mearin ML, Ribes-Koninckx C et al. (2020) European Society Paediatric Gas-troenterology, Hepatology and Nutrition Guidelines for Diagnosing Coeliac Disease 2020. J Pediatr Gastroenterol Nutr. 70, 141-1

Instituto de Salud Pública (ISP), Santiago, Chile. (2016). Recomenda-ciones para la serología en enfermedad celíaca. Accedido en http:// www.ispch.cl/resolucion/1349 el 15 de enero de 2020.

Lerner A. (2014). Serological diagnosis of celiac disease - Moving beyond the tip of the iceberg. International Journal of Celiac Disease 2, 64- 6 .

Lerner A, Jeremias P, Neidhöfer S. \& Matthias T. (2016). Antibodies against neo-epitope $\mathrm{tTg}$ complexed to gliadin are different and more reliable then anti-tTg for the diagnosis of pediatric celiac disease. J Immunol Methods 429,15-20. 


\section{Verbeke et al.}

Lerner A, Ramesh A, Matthias T. (2019). Serologic Diagnosis of Celiac Disease: New Biomarkers. Gastroenterol Clin N Am. 48, 307-317.

McCabe M, Toughill E, Parkhill A, Schell M, Jevic M. \& Nye M. (2012).

Celiac Disease: A medical puzzle. American Journal Nursing 112, 34-44.

Ministerio de Salud Pública (MINSAL) Guía clínica (2015). Búsqueda, Diagnóstico y Tratamiento de la Enfermedad Celíaca. Subsecretaría de Salud Pública. Accedido en https://coacel.cl/sites/default/files/ guia_minsal_enf._celiaca.pdf el 15 de enero de 2020.

Molberg O, McAdam S, Korner M, Quarsten H, Kristiansen C, Madsen $L$, et al. (1998) Tissue transglutaminase selectively modifies gliadin peptides that are recognized by gut-derived T cells in celiac disease. Nature Medicine 4, 713-7.

Pietzak M. (2012). Celiac disease, wheat allergy, and gluten sensiti-vity: when gluten free is not a fad. Journal of Parenteral and Enteral Nutrition 36, 68S-75S.

Porcelli B, Ferretti F, Vindigni C. \& Terzuoli L. (2016). Assessment of a test for the screening and diagnosis of celiac disease. Journal of Clinical Laboratory Analysis 30, 65-70.
Rosenberg O, Lerner A, Pacht A, Grinberg M, Reginashvili D, Henig C, et al. (2012). A novel algorithm for the diagnosis of celiac disease and a comprehensive review of celiac disease diagnostics. Clinical Review in Allergy and Immunology 42, 331-41.

Sapone A, Bai J, Ciacci C, Dolinsek J, Green P, Hadjivassilliou M, et al. (2012). Spectrum of gluten-related disorders: consensus on new nomenclature and classification. BMC Medicine 10,13.

Silvester J, Kurada S, Szwajcer A, Kelly C, Leffler DA \& Duerksen D. (2017). Test for serum transglutaminase and endomysial antibodies do not detect most patients with celiac disease and persistent villous atrophy on gluten-free diet: a meta-analysis. Gastroente-rology 153, 689-701.

Torsten M, Pfeiffer S, Selmi C \& Gershwin M. (2010). Diagnostic Challenges in Celiac Disease and the Role of the Tissue Transglu-taminase-NeoEpitope. Clinical Review in Allergy and Immunology 38, 298-301.

Volta U \& Villanacci V (2011). Celiac Disease: Diagnostic Criteria in Progress. Cellular and Mollecular Immunology 8, 96-102. 